\title{
Revisión
}

\section{La conexión entre la diabetes mellitus y la enfermedad periodontal}

\author{
Michelle Morón Araújo iD $\nabla_{1}$ \\ ${ }^{1}$ Facultad de Odontología, Pontificia Universidad Javeriana, Bogotá, Colombia
}

Cómo citar: Morón-Araujo M. La conexión entre la diabetes mellitus y la enfermedad periodontal. Rev. Colomb. Endocrinol. Diabet. Metab. 2021;8(2):e621. https://doi.org/10.53853/encr.8.2.621

\section{Resumen}

Contexto: existe una relación entre la diabetes y la enfermedad periodontal, siendo la diabetes un factor de riesgo importante para esta afección. Donde para los pacientes con diabetes que también tienen enfermedad periodontal existen efectos adversos sobre el control glucémico.

Objetivo: investigar y analizar la prevalencia de la diabetes mellitus y la relación directa con la enfermedad periodontal. Metodología: se realizó una búsqueda sobre la relación directa de estas dos patologías en las bases de datos Pubmed, Cochrane, Google Scholar y Medline, de contenidos publicados los últimos 10 años, seleccionando solo artículos de revisión.

Resultados y conclusiones: se encontró una relación bidireccional de la diabetes mellitus con la enfermedad periodontal. El odontólogo y el médico deben trabajar en equipo para establecer diagnósticos, planes de prevención y realizar tratamientos para la diabetes mellitus y la enfermedad periodontal, observando su alto impacto mundialmente y la asociación que existe entre estas dos enfermedades.

Palabras clave: diabetes mellitus, enfermedad periodontal, síndrome metabólico, periodontitis, diabetes tipo 2. 


\section{The connection between diabetes mellitus and periodontal disease}

\section{Abstract}

Background: There is a relationship between diabetes and periodontal disease, with diabetes being an important risk factor for this condition. And in those patients with diabetes who also have periodontal disease, there are adverse effects on glycemic control.

Purpose: The objective of this review is to investigate and analyze the prevalence of diabetes mellitus and its direct relationship with periodontal disease.

Methodology: A search was carried out on the direct relationship of these two pathologies in the Pudmed, Cochrane, Google Scholar, Medline databases of the last 10 years, selecting review articles.

Results and conclusions: A bidirectional relationship between diabetes mellitus and periodontal disease was found. The dentist and the doctor must work as a team to establish diagnosis, prevention plans and carry out treatments for diabetes mellitus and periodontal disease, observing their high impact worldwide and the association that exists between these two diseases.

Keywords: Diabetes mellitus, periodontal disease, metabolic syndrome, periodontitis, type 2 diabetes.

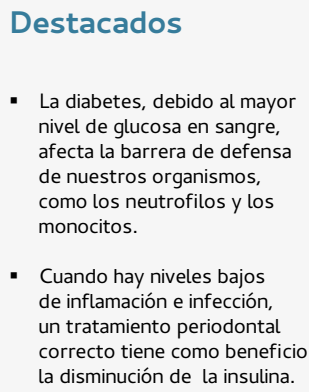

- La diabetes, debido al mayo nivel de glucosa en sangre, afecta la barrera de defensa de nuestros organismos, como los neutrofilos y los monocitos.

- Cuando hay niveles bajos de inflamación e infección, un tratamiento periodontal correcto tiene como beneficio la disminución de la insulina.

\section{Introducción}

La diabetes mellitus (DM) se conoce como un conjunto de enfermedades metabólicas con alteraciones en el metabolismo de los carbohidratos y este conjunto de trastornos metabólicos presenta características principales de hiperglicemia (1).

Este nivel anormalmente elevado de glucosa en sangre es provocado por una deficiencia de insulina causada por el páncreas, causando disfunción celular, resistencia a la insulina en el hígado, tejido muscular o una combinación de ambos. Se sabe que la hiperglucemia crónica conduce a daños a largo plazo de varios órganos y a un mayor riesgo de desarrollar problemas de salud graves que ponen en peligro la vida (1).
La diabetes es una condición con alta prevalencia en el mundo y en Colombia, casi el $7 \%$ de la población de nuestro país ha sido diagnosticada con esta patología y cerca de 17.000 muertes se asocian a ella anualmente. En Colombia, durante 2019 , solo el $51,08 \%$ de los 1,3 millones de pacientes del sistema de salud podían controlar la hemoglobina glucosilada (HbA1c), lo cual constituye una señal preocupante, ya que la falta de control de esta proteína se ha relacionado con otras afecciones, tales como eventos cerebrovasculares (2).

Por suparte, la enfermedad periodontal (EP) es un estado avanzado del proceso inflamatorio gingival que afecta al tejido del sostén dental y está, acompañado de la destrucción del ligamento periodontal, de la pérdida de hueso alveolar y de la 
caída de la dentición. Su etiología es multifactorial y pueden influir factores ambientales, genéticos, susceptibilidad del huésped, etc. (3).

La Organización Mundial de la Salud (OMS) ha descrito que el $99 \%$ de la población mundial padece caries y el $80 \%$ enfermedad periodontal. Se han realizado encuestas de salud bucal (como la Ensab) en Colombia, donde se obtuvieron resultados de todas las formas de enfermedad periodontal (gingivitis y periodontitis) presentes en la población, con una prevalencia de estas de $50,2 \%$ y de esta cifra, el $17,7 \%$ de los casos corresponden a periodontitis de moderada a severa.

En la investigación se ha identificado que a partir de los 20 años las personas empiezan a tener pérdida dental y que el número de dientes que se pierde se incrementa a medida que aumenta la edad. Es así como el 70,4\% de las personas adultas en el país ha perdido algún diente a lo largo de su vida, siendo muy importante señalar que la pérdida es mayor en las mujeres $(73,0 \%)$ frente a los hombres $(67,6 \%)$ y que el $5,2 \%$ de las personas son edéntulos totales (han perdido todos sus dientes), presentándose esto con mayor frecuencia en las personas entre 65 y 79 años $(32,87 \%$ del total de los edéntulos).

Este problema de la salud oral en Colombia se ha convertido en una de las primeras causas de consulta externa, motivo de preocupación dado el impacto que genera la salud oral en la calidad de vida y la relación de la enfermedad periodontal con otras patologías de salud general como la diabetes, enfermedades cardiacas, entre otras $(4,5)$.
La periodontitis y la diabetes son enfermedades crónicas frecuentes, complejas y con una conexión establecida, es decir, la diabetes (especialmente si el control glucémico es deficiente) se asocia con una mayor prevalencia y gravedad de la periodontitis, y la periodontitis grave se asocia con un control glucémico comprometido (5).

La descripción de la diabetes mellitus se remonta a 1500 años antes de Cristo por los egipcios. Luego, en el 400 aC, Sushruta lo describió como orina de miel. En 1869, Langerhans descubrió los islotes en el tejido pancreático. En 1921, Banting y Best describieron sustancias hipoglucemiantes aisladas del páncreas. En 1955 se introdujeron los hipoglucemiantes orales. Gruner informó por primera vez la asociación entre la diabetes y la enfermedad periodontal. En 1928, Williams afirmó que se modifican la gingivitis y la periodontitis entre los pacientes diabéticos, él lo denominó "periodontoclasia diabética". En 1946, Glickman encontró, en un estudio con animales experimentales, que la enfermedad periodontal en los que eran diabéticos no era diferente histológicamente $y$, por lo tanto, no era una entidad clínica única $(6,7)$.

A mediados de la década de 1990, se encontró soporte científico suficiente para la asociación entre la diabetes y la periodontitis, que se comenzó a designar como la sexta complicación de la diabetes. Se han realizado estudios que muestran una mejora tanto en los parámetros clínicos e inmunológicos de la periodontitis como en el control glucémico a largo plazo de la diabetes, tras el tratamiento de la enfermedad periodontal. Además, la evidencia 
científica confirma que un peor control glucémico contribuye a un peor estado periodontal $(7,8)$.

\section{Material y métodos}

Se realizó una búsqueda en las bases de datos de: Pubmed, Cochrane, Google Scholar y Medline. Se incluyó solo la revisión de literatura de los últimos 10 años con las palabras claves: Periodontitis and Diabetes, relation periodontitis and diabetes, diabetes and the relationship with periodontitis. Se obtuvo un total de 832 artículos de todas las bases de datos.

De estos, se incluyeron solo 18 artículos de revisión de la literatura de diferentes idiomas y países para realizar la escritura del artículo y los demás que se encontraron fueron utilizados para complementar este artículo de revisión. Se excluyeron los artículos donde había una desactualización del tema tratado y todos los anteriores al año 2000.

Los tipos de diabetes: diabetes tipo 1, la diabetes tipo 2 y la diabetes gestacional

La diabetes tipo 1 (en el pasado conocida como diabetes insulinodependiente o diabetes juvenil) se define como una afección en la que no se produce insulina como resultado de la destrucción autoinmune de las células $\beta$, productoras de insulina en el páncreas.

La susceptibilidad genética es un factor de riesgo importante en la diabetes tipo 1 y en las personas susceptibles la aparición de la diabetes parece ser provocada por factores ambientales como las infecciones virales y la dieta, en lugar de estar relacionada con factores de estilo de vida. La diabetes tipo 1 suele aparecer en la niñez o en la adultez temprana $(9,10)$.

Por otro lado, la diabetes tipo 2 (anteriormente conocida como diabetes no insulinodependiente o diabetes de inicio en la edad adulta) es el resultado de la resistencia a la insulina: hay una capacidad de respuesta reducida de las células del cuerpo a la insulina, lo que lleva a una capacidad reducida para transferir glucosa fuera de la circulación hacia las células, esto conduce a hiperglucemia (niveles elevados de glucosa en sangre). En las primeras etapas, la secreción de insulina por las células $\beta$ del páncreas puede ser normal pero puede disminuir con el tiempo, lo que da lugar a deficiencia de insulina y a la resistencia de esta $(10,11)$.

La diabetes gestacional es una forma de diabetes que se presenta en mujeres embarazadas sin antecedentes de diabetes que desarrollan hiperglucemia durante el embarazo. Se caracteriza por una reducción de la secreción de insulina y de la resistencia a esta, que por lo general mejora después del embarazo, aunque es posible que una pequeña proporción de las mujeres afectadas queden con diabetes (generalmente del tipo 2) después del embarazo $(11,12)$.

\section{La relación entre la diabetes y la} enfermedad periodontal

Muchos han sido los estudios y los reportes encontrados acerca de la relación entre estas dos patologías. El riesgo y la gravedad de la periodontitis y 
las enfermedades periodontales pueden agravar la resistencia a la insulina y afectar el control glucémico.

Los pacientes con diabetes presentan niveles altos de AGE (Advanced Glycation Endproducts) en tejidos que incluyen los del periodonto, mientras que los diabéticos presentan altos niveles de citoquinas proinflamatorias en suero $y$ líquido del surco gingival.

Además, los monocitos aislados en diabéticos y estimulados con LPS (lipopolisacáridos) secretan mayores concentraciones de citoquinas proinflamatorias y prostaglandinas.

La disglucemia e incluso los niveles de azúcar en sangre ligeramente elevados afectan negativamente la salud bucal y ello se manifiesta en varias enfermedades y afecciones bucales (13).

La enfermedad periodontal, al ser una enfermedad infecciosa, causa inflamación y destrucción del tejido conectivo que soporta al diente, esto se presenta debido a la interacción de las bacterias periodontopatógenas con las células inmunocompetentes y los fibroblastos al provocar la activación y la secreción local de mediadores de la inflamación con carácter catabólico, principalmente TNF- $\alpha$ (factor de necrosis tumoral alfa) y IL-1 (interluquina 1) (14).

Estas infecciones pueden iniciar 0 aumentar la resistencia a la insulina, favoreciendo la respuesta del sistema inmune. Estosfenómenos delainflamación están asociados con el desarrollo de la resistencia a la insulina, la cual está además influenciada por factores ambientales (pero modificados por la genética), como son la escasa actividad física, una alimentación inadecuada, la obesidad o las infecciones (15).

La diabetes puede desarrollar y conducir el control glucémico deficiente, lo que contribuye a una peor condición periodontal por el metabolismo anormal de colágeno como un posible mecanismo involucrado en el desarrollo, disminuyendo la cantidad de colágeno en los tejidos periodontales mediante la reducción de la síntesis de este y el aumento de su degradación $(16,17)$.

La diabetes mellitus induce a una inflamación exacerbada a las bacterias patógenas presentes en la encía, desarrollando una alteración en la capacidad de resolución de la inflamación y en su reparación, produciendo daños periodontales. Estos procesos están mediados por la glicosilación avanzada que se produce, como el desarrollo de la hiperglucemia, y se expresan en los tejidos periodontales de los pacientes con diabetes mellitus.

La diabetes mellitus es posiblemente la única enfermedad sistémica asociada positivamente con la pérdida de inserción clínica en pacientes con periodontitis y su riesgo es de 2,32 veces mayor en pacientes diabéticos no controlados vs. aquellos no diabéticos y diabéticos controlados (18).

La pérdida ósea se presenta con la destrucción tisular, característica de la periodontitis, y afecta al tejido conjuntivo y al hueso alveolar. La degradación del colágeno y de los componentes de la matriz en el tejido conjuntivo se encuentra regulada por procesos inflamatorios en la periodontitis e incluye la producción de diversas metaloproteinasas de la matriz (MPM) (13). 


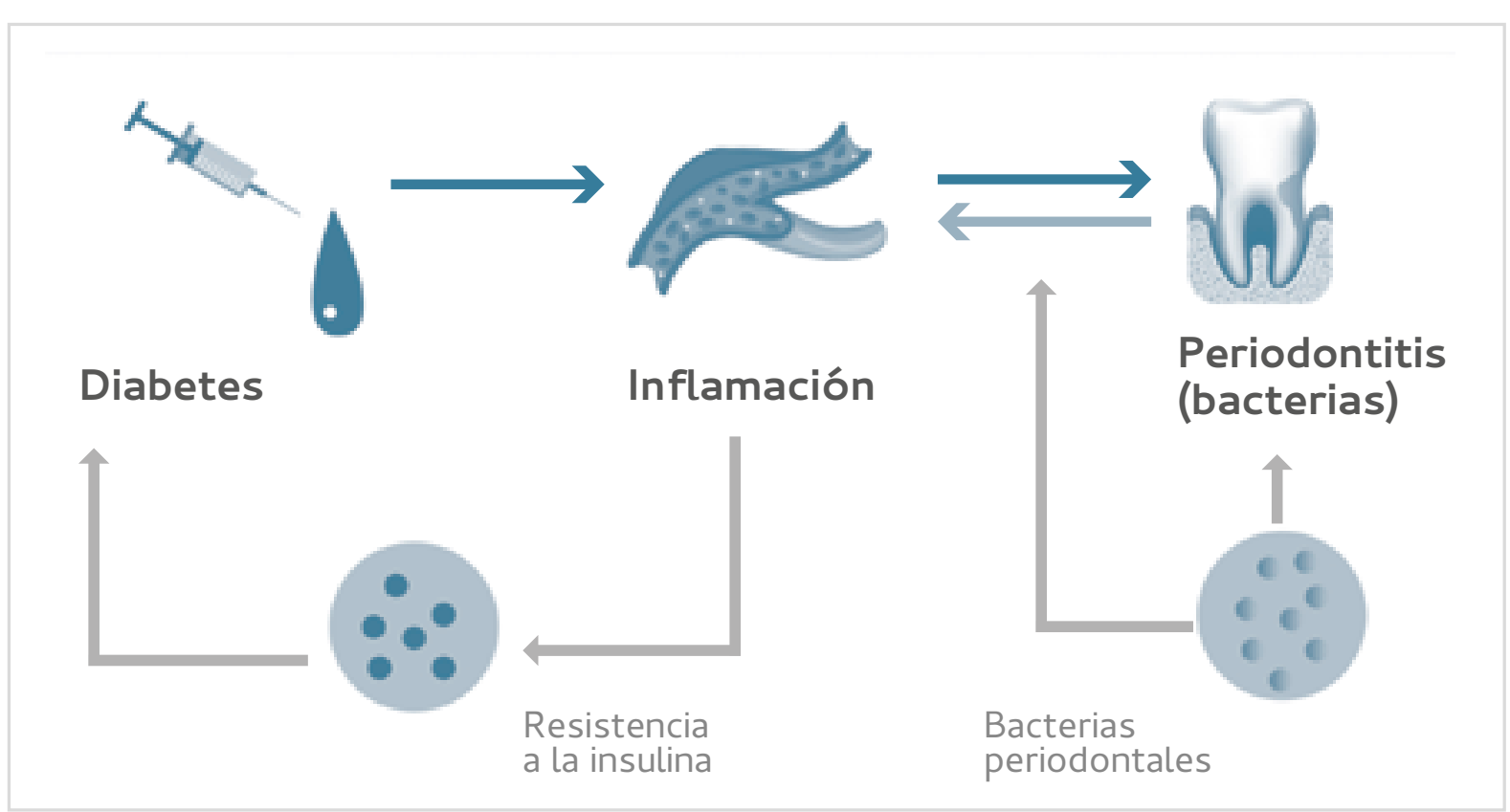

Figura 1. La inflamación y la relación de la diabetes mellitus con la enfermedad periodontal

Fuente: elaboración propia.

Los estudios han demostrado que la diabetes conlleva un riesgo tres veces mayor de periodontitis en comparación con las personas no diabéticas. La pérdida de hueso alveolar aumenta en estos individuos, lo que resulta en una respuesta inflamatoria más persistente en los diabéticos y esto conduce a una mayor pérdida de inserción y alteración de la formación de hueso nuevo (19, 20).

Las complicaciones sistémicas de la diabetes mellitus incluyen: ataque cardiaco, enfermedad renal, microcirculación sanguínea, ceguera y daño a los nervios periféricos. Se encontró en la literatura que más del $90 \%$ de los pacientes diabéticos presentan manifestaciones orales, dado que esta enfermedad daña gravemente los tejidos orales causando enfermedad periodontal, pérdida de dientes, xerostomía, caries, ardor en la boca, disfunción del gusto y de las glándulas salivales, retraso en la cicatrización de heridas, liquen plano, lengua geográfica y candidiasis. Cada vez hay más evidencia sobre una fuerte relación bidireccional entre la diabetes mellitus y la enfermedad periodontal (21).

Numerosos estudios han demostrado una conexión entre la enfermedad periodontal y el control glucémico, teniendo cada enfermedad un impacto potencial sobre la otra $(22,23)$.

La enfermedad periodontal se asocia a un peor control metabólico de la diabetes y es un hecho de observación clínica muy señalado cuando se comparan perfiles glucémicos de diabéticos con enfermedad periodontal, frente a otros que no la padecen. 
La periodontitis puede comenzar o aumentar la resistencia a la insulina de manera similar a como lo hace la obesidad. La inflamación crónica generada por la liberación de estos mediadores de la inflamación está asociada con el desarrollo de la resistencia a la insulina, que está además influenciada por factores ambientales (pero modificados por la genética), como la escasa actividad física, la alimentación inadecuada, la obesidad y las infecciones.

Al ser la enfermedad periodontal un estado inflamatorio crónico, la producción de citoquinas proinflamatorias podría ser el laxo de unión entre la enfermedad periodontal y la diabetes. Cada vez hay más pruebas que apoyan que la inflamación sistémica elevada (biomarcadores de estrés oxidativo y de fase aguda) es resultado de la entrada de organismos periodontales y de sus factores de virulencia en circulación, porcionando plausibilidad biológica para los efectos de la periodontitis en la diabetes. Las interacciones $A G E,-R A G E$ (receptor de $A G E$ ) y las vías mediadas por el estrés oxidativo proporcionan vínculos mecánicos plausibles en la dirección de la diabetes y la periodontitis $(24,25,26)$.

\section{Prevención de la diabetes mellitus y la enfermedad periodontal}

Realizar un buen diagnóstico para establecer la diabetes es importante, algunos síntomas de prediabetes son: aumento de la orina (poliuria), sed (polidipsia), hambre (polifagia), baja de peso inexplicable, visión borrosa, dolores en las extremidades, infecciones constantes, náuseas, vómitos intensos, desmayos y pérdida de la conciencia (27).
El control metabólico es muy importante, ya que se establecen y analizan los parámetros normales de glucosa, la educación diabetológica, la dieta y el ejercicio son importantes para evitar el desarrollo de la diabetes mellitus 2.

Por su parte, el diagnóstico de la enfermedad periodontal se realiza por medio de la historia clínica al conocer y reportar signos, síntomas, estado sistémico, antecedentes familiares, entorno social y realizar la identificación de los factores de riesgo.

En este examen clínico se realiza una inspección del tejido blando, se sondea el surco gingival y se reporta en el periodontograma la magnitud de bolsas, sangrado, movilidad dental, presencia de fístulas, se exploran lesiones de furca y la pérdida de inserción clínica (28).

Adicionalmente, las radiografías periapicales son una herramienta fundamental para el periodoncista, ya que por medio de ellas se evalúan las pérdidas óseas, las lesiones apicales y otras.

Por último, el tratamiento periodontal consta de diferentes fases: de urgencia, sistémica, higiénica, correctiva y de mantenimiento. El objetivo principal de este tratamiento es cambiar la microflora del surco periodontal para devolver la salud a los tejidos de soporte dental. Los hábitos de higiene oral son muy importantes para controlar la salud periodontal, por lo que se deben usar los elementos adecuados como cepillo, hilo dental y enjuague bucal. El correcto cepillado tres veces al día, el cambio del cepillo cada tres meses, asistir a un control odontológico cada seis meses y mantener una alimentación saludable son 
factores importantes para mantener y controlar la salud oral (28).

\section{Resultados y discusión}

Con base en los estudios disponibles, se encontró evidencia y conexión entre la diabetes y la enfermedad periodontal, sin embargo, el número de estudios de cohortes es limitado. Se necesitan más estudios futuros de cohortes bien diseñados para apoyar la conexión entre estas dos enfermedades.

\section{Conclusión}

La atención interprofesional tiene como objetivo contribuir a mejorar la salud, el bienestar y la calidad de vida de las personas con diabetes mellitus y con enfermedad periodontal. Por lo tanto, la atención a las enfermedades bucodentales infecciosas y la derivación a los profesionales de la salud oral para su tratamiento pueden ser una herramienta importante para los profesionales de la atención médica en la prevención y el tratamiento de la diabetes mellitus.

Lainterrelaciónentreambas afecciones deja constancia de la importancia de la necesidad de una buena comunicación entre el médico y el odontólogo de los pacientes diabéticos, teniendo siempre en cuenta la posibilidad de que ambas enfermedades puedan estar ocurriendo simultáneamente.

El control de la enfermedad periodontal puede mejorar el control glucémico en pacientes con diabetes mellitus. A su vez, un mejor control glucémico puede contribuir a un mejor control de la enfermedad periodontal.

\section{Declaración de fuentes de financiación}

Esta investigación no recibió ningún soporte financiero.

\section{Conflicto de intereses}

La autora declara que no tuvo ningún conflicto de interés asociado al desarrollo de la investigación.

\section{Referencias}

[1] Asociación Colombiana de Endocrinología [Internet]. Diabetes y Covid-19: Cuidados especiales para personas con diabetes en época de coronavirus. Bogotá D. C.: ACE; 2020 [citado 2020 ag, 24]. Disponible en: https://www. endocrino.org.co/ w p-content/uploads/2020/03/RecomendacionesACE-sobre-Diabetes-y-Covid-19.p

[2] Hospital Universitario San Ignacio [Internet]. La Diabetes, la causa de 17 mil muertes en Colombia. Bogotá: Hospital Universitario San Ignacio; 2019 [citado 2020 ag. 24]. Disponible en: http://www.husi. org.co/el-husi-hoy/la-diabetesla-causa-de-17-mil-muertes-encolombia 
[3] Preshaw P, Bissett S. Periodontitis and Diabetes. $\mathrm{Br}$ Dent. 2019;227(7):577-84. https://doi. org/ 10.1038/s41415-019-0794-5

[4] Pardo F, Hernández L. Enfermedad periodontal: enfoques epidemiológicos para su análisis como problema de salud pública. Rev Sal Pública. 2018;20(2):25864. https://doi.org/ 10.15446/rsap. v20n2.64654

[5] Ministerio de la Protección Social. Resolución Número 3577 De 2006, por la cual se adopta el Plan Nacional de Salud Bucal. 2006. p. 1-15.

[6] Borgnakke W. IDF Diabetes Atlas: Diabetes and oral health A two-way relationship of clinical importance. Diabetes Res Clin Pract. 2019;(157):1-19. https://doi. org/ 10.1016/j.diabres.2019.107839

[7] Rajkumar D, Subramanium G, Natarajan S, Mahalingam L, Thangavelu K. Diabetes and periodontal disease. J Pharm Bioallied Sci. 2012; (supl. 2): S280-2.

[8] Pihlstrom BL, Michalowicz BS, Johnson NW. Periodontal diseases. Lancet 2005;366:1809-20. https:// doi.org/10.1016/S01406736(05)67728-8

[9] Casanova L, Hughes F, Preshaw P. Diabetes and periodontal disease: a two-way relationship. $\mathrm{Br}$ Dent J. 2014;(217):433-7. https://doi. org/ 10.1038/sj.bdj.2014.907

[10] Negrato C, Tarzia O, Jovanovič L, Montenegro L. Periodontal disease and diabetes mellitus. J Appl Oral
Sci. 2013;21(1):1-12. https://doi. org/10.1590/1678-7757201302106

[11] Bascones A, Matesanz P, Bermejo M, González M, Bascones J, Meurman J. Periodontal disease and diabetes-Review of the Literature. Med Oral Patol Oral Cir Bucal. 2011;16(6):e722-9. https://doi. org/ 10.4317/medoral.17032

[12] Manzon J, Caramello C. Diabetes $y$ enfermedad periodontal, una relación de doble vía. Rev facultad odont. 2018;1(11):1-5. https:// doi.org/ 10.30972/rfo.1113864

[13] Lindhe L. Periodoncia clínica e implantología odontológica. Buenos Aires: Editorial Médica Panamericana; 2011. p 486-487.

[14] Gursoy U, Yildiz CS, Kononen E, Gursoy M, Dogan B. Salivary interleukin-17 and tumor necrosis factor-alpha inrelation to periodontitis and glycemic status in type 2 diabetes mellitus. J Diab. 2015;7:681-8. https://doi. org/ 10.1111/ 1753-0407.12228

[15] Herrera D, Rodríguez-Casanovas HJ, Herrera-Pombo JL. Diabetes $y$ enfermedades periodontales [Internet]. Madrid: SEPA/Fundación SED [citado 2016 en. 26]. 8 p. Disponible en: https://www.sepa. es/images/stories/SEPA/ESTA R al_DIA/DIABETES/ Dossier_ DIĀBETES.pdf

[16] Graves D, Ding Z, Yingming Y. The impact of diabetes on periodontal diseases. Periodontol 2000. 2020;82(1):214-24. https://doi. org/ $10.1111 /$ prd.12318 
[17] Castillo G, López R, Tineo M, Villarreal N, Alarcón M. Diabetes mellitus y enfermedad periodontal: Revisión bibliográfica de la situación actual. Rev Estomatol Herediana. 2012;22(3):183-8. https://doi. org/ 10.20453/reh.v22i3.125

[18] Viaña-Bermúdez FA, ErazoCoronado AM, Crescente-Pertuz NA, Mustafa-Awadallah A, RugelesEstupiñan C. Factores influyentes en pacientes con periodontitis y diabetes mellitus tipo II. Salud Uninorte. 2021;37(1),3851. https://doi.org/ 10.14482/ sun.37.1.616.46

[19] Abdalrahman R, Gismalla B. The impact of chronic periodontitis on serum tumor necrosis factor- $\alpha$ and HbA1c levels in type 2 diabetic patients. Saudi J Health Sci. 2021;10(1):7-13. https://doi. org/10.4103/sjhs.sjhs_224_20

[20] Bascones A, Martínez M, Boscones J. Diabetes and periodontitis: $A$ bidirectional relationship. Med Clin. 2015;145(1):31-5. https://doi. org/ 10.1016/j.medcli.2014.07.019

[21] Nazir M, Alghamdi L, Al-Kadi M, AlBeajan N, Alrashoudi L, Alhussan $M$. The burden of Diabetes, Its Oral Complications and Their Prevention and Management. Maced J Med Sci. 2018;6(8):1545-53. https://doi . org/10.3889/oamjms.2018.294

[22] Genco R, Graziani F, Hasturk $H$. Effects of periodontal disease on glycemic control, complications, and incidence of diabetes mellitus. Periodontol 2000. 2020;83(1):5965. https://doi.org/ 10.1111/prd.12271
[23] Stanko P, Izakovicova L. Bidirectional association between diabetes mellitus and inflammatory periodontal disease. A review. Biomed Pap Med Fac Univ Palacky Olomouc Czech Repub. 2014;158(1):35-8. https://doi. org/ 10.5507/bp.2014.005

[24] Wu C, Yuan Y, Liu H, Li S, Zhang $\mathrm{B}$, Chen W, et al. Relación epidemiológica entre periodontitis y diabetes mellitus tipo 2. BMC Oral Health. 2020;20(204). https://doi. org/ 10.1186/s12903-020-01180-w

[25] Qayum B, Syed A, Izhar F, Ali A. Bidirectional relationship between diabetes and periodontal disease: review of evidence. J Pak Med Assoc. 2010;60(9):766-8.

[26] Lee CY, Kuan YH, Tsai YF, Tai CH, Tsai $\mathrm{TH}$, Huang $\mathrm{KH}$. Correlation between Diabetes Mellitus and Periodontitis in Taiwan: A Nationwide Cohort Study. Diabetes Res Clin Pract. 2021;150:245$52 . \quad$ https://doi.org/ 10.1016/j. diabres.2019.03.019

[27] Choptian-Fiorese IF, CamargoGomes J, Custódio-dos-Santos BC, Wachter F, Piana EA, CostaBrancalhão RM, et al. Effects of the Association of Periodontitis and Type 1 Diabetes Mellitus Induced on Periodontal Tissues and the Duodenal Mucosa of Wistar Rats. Inflammation. 2021;44(2):70413. https://doi.org/ 10.1007/s10753020-01369-9

[28] OMS. https://www.who.int/ diabetes/action_online/ basics/es/ index1.html 
[29] Ferro M, Gómez M. Fundamentos de la odontología periodoncia. Bogotá: Editorial Javeriana; 2007.

[30] Mealey BL, Oates TW. American Academy of Periodontology. Diabetes mellitus and periodontal diseases. J Periodontol .2006;77:1289-303. https://doi. org/ 10.1902/ jop.2006.050459

[31] Faria R, López A, Rodríguez $H$, Herrera D. Efectos de las enfermedades periodontales sobre la diabetes. Av Diabetol. 2013;29(5):151-9. https://doi. org/ 10.1016/ j.avdiab.2013.07.003

[32] Ide R, Hoshuyama T, Wilson D, Takahashi K, Higashi T. Periodontal disease and incident diabetes: a seven-year study. J Dent Res. 2011;90:41-6. https://doi. org/ 10.1177/ 0022034510381902

[33] Morita I, Inagaki K, Nakamura F, Noguchi T, Matsubara T, Yoshii $\mathrm{S}$, et al. Relationship between periodontal status and levels of glycated hemoglobin. J Dent Res. 2012;91:161-6. https://doi. org/ 10.1177/0022034511431583

[34] Borgnakke WS, Ylostalo PV, Taylor GW, Genco RJ. Effect of periodontal disease on diabetes: Systematic review of epidemiologic observational evidence. J Clin Periodontol. 2013;40(supl. 14):S135-52. https://doi org/ $10.1111 /$ jcpe.12080

[35] Reyes L, Herrera D, Kozarov E, Roldan S, Progulske A. Periodontal bacterial invasion and infection: Contribution to atherosclerotic pathology. J Clin Periodontol. 2013;40(supl. 14):S30-50. https:// doi.org/ 10.1111/jcpe.12079

[36] Bastos AS, Graves DT, Loureiro AP, Rossa-Junior C, Abdalla DS, FaulinTdo $\mathrm{E}$, et al. Lipid peroxidation is associated with the severity of periodontal disease and local inflammatory marker in patients with type 2 diabetes. J Clin Endocrinol Metab. 2012;97:E1353-62. https://doi.org/ 10.1210/jc.20113397

[37] Scannapieco FA, Dasanayake AP, Chhun N. Does periodontal therapy reduce the risk for systemic diseases? Dent Clin North Am. 2010;54:163-81. https://doi. org/10.1016/j.cden.2009.10.002

[38] Koromantzos PA, Makrilakis K, Dereka X, Katsilambros N, Vrotsos IA, Madianos PN. A randomized, controlled trial on the effect of non-surgical periodontal therapy in patients with type 2 diabetes. Part I: Effect on periodontal status and glycaemic control. J Clin Periodontol. 2011;38:1427. https://doi.org/10.1111/j.1600051X.2010.01652.x

[39] Engebretson SP, Hey-Hadavi J. Sub-antimicrobial doxycycline for periodontitis reduces hemoglobin Alc in subjects with type 2 diabetes: a pilot study. Pharmacol Res. 2011;64:624-9. https://doi. org/10.1016/j.phrs.2011.06.024

[40] Sun WL, Chen LL, Zhang SZ, Ren $Y Z$, Qin GM. Changes of adiponectin and inflammatory cytokines after periodontal intervention in type 2 diabetes patients with periodontitis. 
Arch Oral Biol. 2010;55:970$4 . \quad$ https://doi.org/10.1016/j. archoral bio.2010.08.001

[41] Munenaga Y, Yamashina T, Tanaka J, Nishimura F. Improvement of glycated hemoglobin in Japanese subjects with type 2 diabetes by resolution of periodontal inflammation using adjunct topical antibiotics: Results from the Hiroshima Study. Diabetes Res Clin Pract. 2013;100:5360 . https://doi.org/10.1016/j. diabres.2013.01.028 